\title{
Birth weight, BMI in adulthood and latent autoimmune diabetes in adults: A Mendelian
} \section{randomization study}

Yuxia Wei, MSc, ${ }^{{ }^{*}}$ Yiqiang Zhan, PhD, ${ }^{1}$ Josefin E. Löfvenborg, PhD, ${ }^{1}$ Tiinamaija Tuomi, MD, ${ }^{2}$ Sofia Carlsson, $P h D^{1}$

1. Institute of Environmental Medicine, Karolinska Institutet, Stockholm, Sweden.

2. Department of Endocrinology, Helsinki University Hospital, Helsinki, Finland; Institute for Molecular Medicine Finland FIMM and Research Programs Unit, Diabetes and Obesity, University of Helsinki, Helsinki, Finland; Folkhalsan Research Center, Helsinki, Finland; Lund University, Malmö, Sweden.

\section{*Corresponding author}

Yuxia Wei, MSc

Institute of Environmental Medicine,

Karolinska Institutet

Nobels väg 13, 17177 Stockholm, Sweden

Phone: +46769266173

Email: yuxia.wei@ki.se

YZ: yiqiang.zhan@ki.se

JEL: josefin.lofvenborg@ki.se

TT: tiinamaija.tuomi@hus.fi

SC: sofia.carlsson@ki.se

Word count: Abstract 256, Main text 3197 


\begin{abstract}
Aims: Observational studies have found an increased risk of latent autoimmune diabetes in adults (LADA) associated with low birth weight and adult overweight/obesity. We aimed to investigate whether these associations are causal, using a two-sample Mendelian randomization (MR) design. In addition, we wanted to compare results for LADA and type 2 diabetes.
\end{abstract}

Methods: We identified 129 SNPs as instrumental variables (IVs) for birth weight from a genome-wide association study (GWAS) of the Early Growth Genetics Consortium (EGG) and the UK Biobank. We identified 820 SNPs as IVs for adult BMI from a GWAS of the UK Biobank and the Genetic Investigation of ANthropometric Traits consortium (GIANT). Summary statistics for the associations between IVs and LADA were extracted from the only GWAS involving 2,634 cases and 5,947 population controls. We used the inverse-variance weighted (IVW) estimator as our primary analysis, supplemented by a series of sensitivity analyses.

Results: Genetically determined birth weight was inversely associated with LADA (OR per SD [ 500 g] decrease in birth weight: $2.02,95 \%$ CI: $1.37-2.97)$. In contrast, genetically predicted BMI in adulthood was positively associated with LADA (OR per SD $\left[\sim 4.8 \mathrm{~kg} / \mathrm{m}^{2}\right]$ increase in BMI: 1.40, 95\% CI: 1.14-1.71). Results persisted in a range of sensitivity analyses using other MR estimators or excluding some IVs. With respect to type 2 diabetes, the association with birth weight was not stronger than in LADA while the association with adult BMI was stronger than in LADA.

Conclusions/interpretation: This study provides genetic support for a causal link between low birth weight, adult overweight/obesity, and LADA.

Keywords: Genetics / Epidemiology; Human; Weight regulation and obesity 


\section{Research in context}

\section{- What is already known about this subject? (maximum of 3 bullet points)}

Previous Mendelian randomization studies have found that both lower birth weight and higher adult BMI are associated with an increased risk of type 2 diabetes. In contrast, the evidence on latent autoimmune diabetes in adults (LADA) is very limited. Only a few observational studies indicated an inverse association between birth weight and LADA, or a positive association between adult BMI and LADA.

- What is the key question?

Are birth weight and adult adiposity causally associated with LADA?

- What are the new findings?

Genetically determined birth weight was inversely associated with LADA while genetically predicted BMI in adulthood was positively associated with LADA.

The association with birth weight was not weaker for LADA than for type 2 diabetes, while adult BMI had a greater impact on type 2 diabetes than on LADA.

\section{- How might this impact on clinical practice in the foreseeable future?}

Findings from this study indicate that measures should be taken to reduce the prevalence of adult overweight/obesity for the prevention of diabetes with and without an autoimmune component. The mechanism linking low birth weight to diabetes remains to be explored.

\section{Abbreviations}

DIAGRAM: the DIAbetes Genetics Replication And Meta-analysis Consortium; EGG: the Early Growth Genetics Consortium; GIANT: the Genetic Investigation of ANthropometric Traits consortium; GWAS: genome-wide association study; InSIDE: the instrument strength is independent of instrument's direct effect; IV: instrumental variable; IVW: inverse-variance weighted; LADA: latent autoimmune diabetes in adults; LADY, Latent autoimmune diabetes 
medRxiv preprint doi: https://doi.org/10.1101/2021.10.25.21265464; this version posted October 25, 2021. The copyright holder for this preprint (which was not certified by peer review) is the author/funder, who has granted medRxiv a license to display the preprint in perpetuity.

in the young; MR: Mendelian randomization; MR- Egger: Egger regression of Mendelian randomization; MR-PRESSO: the Mendelian randomization pleiotropy residual sum and outlier approach. 


\section{Introduction}

Latent autoimmune diabetes in adults (LADA) is a hybrid form of diabetes. Genetically, it is closely related to type 1 diabetes with a strong link to HLA genotypes[1,2], while many of its clinical features such as metabolic syndrome are shared with type 2 diabetes[2]. LADA is characterized by pancreatic autoantibodies, slow progression to insulin dependence and is usually restricted to adult patients[2] although a similar phenomenon has been described in younger patients (LADY, Latent autoimmune diabetes in the young)[3]. Autoantibody testing is required to separate LADA from type 2 diabetes. Around 5-14\% of adult patients diagnosed with "type 2 diabetes" in Europe, North America, and Asia have pancreatic autoantibodies[2].

Risk factors of type 2 diabetes are extensively studied[4, 5] and the disease can be prevented or postponed by maintaining a healthy weight and physical activity[6]. In comparison, the evidence on environmental/lifestyle risk factors of LADA is limited[7]. However, we have previously reported on an increased risk of LADA in relation to overweight/obesity [8] and low birth weight[9], in line with findings in type 2 diabetes[10, 11]. Observational studies are prone to residual confounding and reverse causation, while randomized control trials may be unfeasible in studying some risk factors of diseases. Individuals' genotypes are randomly assigned from their parents before conception and thus are less likely to suffer from confounding or reverse causation[12]. Taking advantage of the natural experiments, Mendelian randomization (MR) studies use genetic variants as instrumental variables (IVs) for an environmentally modifiable exposure to make causal inference about the outcome[12].

Our aim was to investigate whether low birth weight and adult adiposity are implicated in the etiology of LADA by, for the first time, using a two-sample MR design. In addition, we wanted to compare these associations in LADA and type 2 diabetes. 


\section{Methods}

\section{Study design}

This was a two-sample MR study using summary statistics from two separate genome-wide association studies (GWAS) of non-overlapping samples of the same underlying population; one provided measures of the associations between IVs and the exposure and the other on the associations between IVs and the outcome[13].

\section{Genetic instruments}

\section{Birth weight}

Summary statistics ( $\beta$ coefficients and standard errors) for the associations between IVs and birth weight were extracted from the most recent meta-analysis of the Early Growth Genetics Consortium (EGG) and UK Biobank[14]. Information on birth weight had been collected by measurement at birth, obstetric records, medical registers, interviews with the mother, or selfreport as adults in different included studies (eTable 1). The GWAS identified 145 independent SNPs associated with own birth weight at $P<6.6 \times 10^{-9}$ (genome-wide significance threshold revised by authors of the GWAS) in 298,140 European individuals. Among the 145 SNPs, 129 SNPs (eTable 2) were available in the GWAS dataset of LADA described below, explaining $2.61 \%$ of the variance in birth weight.

\section{Adult BMI}

The primary set of IVs for BMI in adulthood was obtained from a meta-analysis including 681,275 individuals of European ancestry from UK Biobank and the Genetic Investigation of ANthropometric Traits (GIANT) consortium[15]. Information on BMI had mainly been obtained through clinical measurements (eTable 1). SNPs of this meta-analysis have been used as IVs for BMI in a previous MR study[16]. In the meta-analysis, independent SNPs were selected through LD clumping, in which SNPs with LD measure of $r^{2}>0.01$ within a 
10000-kb window were pruned, resulting in a total of 839 independent SNPs with $P<1 \times$ $10^{-8}$ (genome-wide significance threshold revised by authors of the meta-analysis). Of these, 817 SNPs were available in the GWAS of LADA and proxy SNPs in linkage disequilibrium $\left(\mathrm{LD} ; \mathrm{r}^{2}>0.8\right)$ with three of the unavailable SNPs were identified using LDlink[17]. The 820 SNPs explained $7.43 \%$ of the variance in adult BMI (eTable 3).

The GIANT consortium had samples overlapping with the GWAS of type 2 diabetes (described below). Therefore, we selected a secondary set of IVs (734 independent SNPs) for adult BMI exclusively from the UK Biobank study (https://gwas.mrcieu.ac.uk/) when comparing the BMI-LADA association with the BMI-type 2 diabetes association. The 734 SNPs explained $7.86 \%$ of the variance in adult BMI.

\section{GWAS of LADA}

LADA was the primary outcome in the present study. We obtained summary statistics for the association between the abovementioned SNPs and LADA from the only GWAS of LADA hitherto. It included 2,634 LADA cases and 5,947 population controls of European ancestry from Sweden, Denmark, Germany, and the UK, and the analysis was adjusted for sex and principal components (to correct for potential bias due to population structure)[1]. LADA was defined based on: (1) adult-onset (age at diagnosis $>20,30$, or 35 years); (2) the presence of diabetes-associated autoimmune autoantibodies, in particular glutamic acid decarboxylase autoantibody (GADA) positivity; and (3) lack of insulin requirement for 6 months or 1 year after diagnosis[1].

\section{GWAS of type 2 diabetes}

Summary statistics for the SNP-type 2 diabetes association were obtained from the

DIAGRAM (DIAbetes Genetics Replication And Meta-analysis) Consortium, which included 26,676 type 2 diabetes cases and 132,532 controls of European ancestry[18]. Summary statistics in this study were adjusted for age, sex, and principal components[18]. 


\section{Data Harmonization}

We checked the effect allele, reference allele, and effect allele frequency in the GWAS datasets. The $\beta$ coefficient for the SNP-exposure association was "flipped" if the effect allele for the SNP-exposure association was the reference allele for the SNP-outcome association. We paid attention to palindromic (A/T or $\mathrm{C} / \mathrm{G}) \mathrm{SNPs}$ and there is no ambiguity in matching effect alleles for these SNPs between GWAS datasets of the exposure and the outcome.

\section{Statistical analysis}

We measured instrument strength of each SNP using F statistic[19], which equals the $\beta$ coefficient for the SNP-exposure association divided by the square of the standard error for the $\beta$ coefficient. A larger F statistic indicates stronger instrument strength[19]. Birth weight and adult BMI were all inverse-normally transformed and analyzed in an additive model in the GWAS. Therefore, the risk estimates based directly on the summary statistics were ORs and $95 \%$ CIs for LADA and type 2 diabetes per SD change in exposures.

\section{Main analysis}

The inverse-variance weighted (IVW) method was used to assess the risk of LADA in relation to birth weight based on 129 IVs and adult BMI based on 820 primary IVs. The IVW method can be fitted in weighted linear regression[20, 21]. It provides a more precise risk estimate than other methods when all the IVs are valid[22].

\section{Sensitivity analyses}

Central assumptions in an MR study are that an IV only affects the outcome through the exposure, not through a direct pathway to the outcome or via a confounder (eFigure 1)[23], otherwise there will be directional pleiotropy and the IV is invalid.

The IVW estimator used in the main analyses assumes that all the IVs are valid[22]. Several sensitivity analyses using other MR estimators were conducted to test the robustness of the 
results based on the IVW estimator. Some of them can detect potential directional pleiotropy, and some of them do not require all IVs to be valid. First we used the robust IVW method which replaces the standard linear regression in the IVW method with a robust regression[24]. This method has a greater power than IVW to reject causal null hypothesis when there is balanced pleiotropy (average pleiotropic effect: 0) and the instrument strength is independent of instrument's direct effect (InSIDE[25] assumption) [22]. Furthermore, the weighted median method was used, which provides consistent estimates when $>50 \%$ of IVs are valid and does not rely on the InSIDE assumption[26]. MR-Egger (Egger regression of Mendelian randomization) can give a causal estimate under the InSIDE[25] assumption even when all the IVs are invalid, with the slope coefficient of the regression model representing the logarithmic OR[25]. This method indicates overall directional pleiotropy[25] if the estimated intercept term in the regression model is non-zero[25]. Finally, the MR-PRESSO (the Mendelian randomization pleiotropy residual sum and outlier approach) estimator was used. MRPRESSO is based on the IVW method and detects outliers (potential pleiotropic SNPs), produces corrected OR by removing outliers, and evaluates distortion of risk estimate by outliers [27].

In the present study, we further excluded outliers detected by MR-PRESSO from the IVs, and then re-analyzed the data using all different MR estimators including the IVW estimator.

We did other sensitivity analyses based on the IVW method by excluding some SNPs from the 129 IVs for birth weight and 820 primary IVs for adult BMI. These sensitivity analyses included several conservative analyses to minimize the possibility that the IVs affect the outcome through a pathway outside the exposure, and a leave-one-out analysis to investigate whether the association in the main analysis would disappear by excluding any one of the IVs. In the analysis of birth weight, conservative analysis 1 only included SNPs with fetal-only effects as IVs, to minimize the possibility that IVs affect LADA through maternal 
confounding factors; Conservative analysis 2 further excluded SNPs associated with diabetesrelated traits at nominal significance level (Bonferroni-corrected), to minimize the possibility that IVs affect LADA directly; We further excluded SNPs associated with any trait (except birth weight) at genome-wide significance (conservative analysis 3), and SNPs associated with lifestyle factors (conservative analysis 4) and adult body size (conservative analysis 5) at nominal significance level (Bonferroni-corrected). In the analysis of adult BMI, we excluded SNPs associated with diabetes-related traits at nominal significance level (Bonferronicorrected) or any trait (except adult body size) at genome-wide significance (conservative analysis 1), SNPs associated with lifestyle factors at nominal significance level (Bonferronicorrected, conservative analysis 2), and SNPs associated with birth weight at nominal significance level (Bonferroni-corrected, conservative analysis 3). More details about conservative analyses are provided in eFigure 2 and eTable 4.

\section{Comparison between LADA and type 2 diabetes}

For comparison between LADA and type 2 diabetes, we used the same 129 IVs for birth weight and 734 secondary IVs for adult BMI in the analyses, to ensure the comparability of results. The associations of birth weight and adult BMI with type 2 diabetes were assessed using all the different MR estimators described above. Outlier-corrected ORs (95\% CIs) from MR-PRESSO were used for comparison between LADA and T2D if MR-PRESSO detected outliers, otherwise the results of IVW were used for comparison.

MR analysis was conducted using MendelianRandomization and MR-PRESSO package in R 4.0.4. All statistical tests were two-sided, with $P<0.05$ indicating statistical significance.

\section{Ethical approval}

Our study only used GWAS summary statistics, and no individual-level data were used. An ethical permit is not required according to Karolinska Institutet. 


\section{Results}

A flow chart of the study design is provided in Figure 1. The SD of birth weight and BMI in adulthood was approximately $500 \mathrm{~g}$ and $4.8 \mathrm{~kg} / \mathrm{m}^{2}$, respectively (eTable 1). F statistics for IVs were all above 10 in the present study (eTable 2 and eTable 3).

\section{Birth weight and LADA}

Genetically determined birth weight based on 129 IVs was inversely associated with LADA (eFigure 3). In the main analysis, the OR of LADA was 2.02 (95\% CI: 1.37-2.97) for each SD decrease in birth weight (Figure 2).

The inverse association between birth weight and LADA was also observed using other MR estimators (Figure 2). MR-Egger indicated no directional pleiotropy (intercept: $-0.012, P$ for directional pleiotropy: 0.443). MR-PRESSO detected the SNP rs3184504 as the outlier and the outlier-corrected OR was 1.83 (95\% CI: 1.29-2.59). No distortion in estimate by the outlier ( $P$ : 0.457) was detected in MR-PRESSO. After excluding rs3184504 from the IVs, results from different MR estimators also supported an inverse association between birth weight and LADA, and no directional pleiotropy was detected based on the 128 remaining IVs (eFigure 4).

The inverse association between birth weight and LADA was also observed in a series of conservative analyses by restricting IVs to SNPs with fetal-only effects, by further excluding SNPs associated with diabetes-related traits at $P<0.05 / 129$, by further excluding SNPs associated with any other trait at $P<5 \times 10^{-8}$, and by further excluding SNPs associated with lifestyle factors or adult BMI at $P<0.05 / 129$ (eTable 5). Leaving one of the 129 SNPs out each time did not change the direction of association (eFigure 5). 


\section{Adult BMI and LADA}

Genetically determined adult BMI based on the 820 primary IVs was positively associated with LADA (eFigure 6). One SD increase in adult BMI was associated with an OR of 1.40 (95\% CI: 1.14-1.71) for LADA using the IVW method (Figure 3).

MR-Egger indicated no directional pleiotropy (intercept $=-0.007, P$ for directional pleiotropy: 0.119) while MR-PRESSO detected two outliers, rs11066188 and rs10840606. Outliers did not distort the results ( $P$ for distortion test: 0.777$)$ and the outlier-corrected OR was similar to the OR obtained with IVW. Robust IVW and weighted median also showed results comparable to those estimated by IVW (Figure 3). After excluding rs11066188 and rs10840606 from the IVs, no major change in results from different MR estimators was observed (eFigure 7).

All the three conservative analyses (eTable 5) and the leave-one-out analysis (data not shown) showed similar results with the main analysis.

\section{Comparison between LADA and type 2 diabetes}

When assessing the association between birth weight and type 2 diabetes, MR-PRESSO detected 10 outliers and indicated distortion by outliers ( $P$ for distortion: 0.003 , eTable 6). After correction for outliers, the OR of type 2 diabetes in relation to one SD decrease in genetically determined birth weight was 1.45 (95\% CI: 1.25-1.70), which was slightly smaller than the magnitude of the birth weight-LADA association (Table 1).

MR-PRESSO detected 9 outliers when assessing the association between adult BMI and T2D based on the 734 secondary IVs (eTable 7). The OR of type 2 diabetes was 2.33 (95\% CI: 2.16-2.51) for each SD increase in adult BMI after outlier removal (Table 1 and eTable 7). For LADA, the same IVs yielded an outlier-corrected OR of 1.42 (95\% CI: 1.18-1.72) (Table 
1, eTable 7) which was similar to the association between LADA and adult BMI observed based on the 820 primary IVs.

\section{Discussion}

\section{Main findings}

This study provides genetic evidence that low birth weight and adult adiposity confer an increased risk of LADA. We also confirm findings of previous MR studies in showing that lower birth weight[28-30] and higher BMI[31-38] in adulthood are associated with increased risk of type 2 diabetes. To the best of our knowledge, this is the first MR study to explore the role of environmental/lifestyle factors in the etiology of LADA.

\section{Main findings in relation to previous studies}

The results regarding birth weight are in line with those of our previous observational study indicating a two-fold increased risk of LADA in individuals with a birth weight $<3 \mathrm{~kg}$ compared to $\geq 4 \mathrm{~kg}[9]$. Notably, the association with birth weight was not weaker for LADA than for type 2 diabetes which is also in line with previous observational data[9]. The mechanism linking birth weight to LADA and type 2 diabetes remains unclear. The Barker hypothesis proposes that an adverse intrauterine environment leads to both lower birth weight and higher risk of future cardiometabolic risk[39]. However, we restricted IVs to SNPs with fetal-only effects and there was no major change in OR. Therefore, the inverse association observed in the present study may not be explained by pleiotropy introduced by the intrauterine environment. The fetal insulin hypothesis proposes that genetically determined insulin resistance in the fetus results in impaired insulin-mediated fetal growth as well as insulin resistance in adult life[40]. However, our findings do not support this hypothesis, since the association between birth weight and LADA did not attenuate after excluding SNPs associated with diabetes-related traits (including insulin resistance). Our findings suggest a 
direct effect of low birth weight on the risk of LADA, but the underlying mechanism remains to be explored.

Findings regarding LADA and adult BMI were also in line with previous observational data[8]. Overweight and obesity are strongly associated with development of insulin resistance[41] and this may explain a causal link between adult adiposity and LADA. In support hereof, a positive association between BMI and insulin resistance was observed in LADA patients[8]. The association with BMI was stronger for type 2 diabetes than LADA, which is in line with previous findings[8]. This is to be expected since insulin resistance tends to be less pronounced in LADA compared to type 2 diabetes patients [2, 8, 42]. A previous MR study also found support for a link between childhood adiposity and type 1 diabetes[43]. This implies that overweight/obesity is implicated in the promotion of all major types of diabetes and emphasizes that it is crucial to prevent overweight in order to reduce the incidence of diabetes.

\section{Assessment of MR assumptions}

A major concern in MR studies is the violation of IV assumptions. These assumptions cannot be fully tested, but we used several approaches to minimize this potential bias. First, we applied different MR estimators, some of which detected and corrected for potential directional pleiotropy from statistical perspective. There was no major change in ORs after excluding outliers, indicating the robustness of the results. Second, we excluded some SNPs in several conservative analyses, in which the associations of birth weight and adult BMI with LADA persisted. It should be noted that excluding these SNPs in the conservative analyses because of suggested pleiotropic effects in observational studies, does not prove that they are in fact invalid SNPs. 


\section{Strengths and limitations}

There are several strengths in the present study. First, the two-sample MR design using genetic variants as unbiased proxy minimizes confounding and reverse causation. Second, the application of different MR estimators and a series of conservative analyses reduces the risk of bias caused by directional pleiotropy. Third, we confirmed findings from previous studies on type 2 diabetes and used type 2 diabetes as a "positive control" to show that the instruments and methods used for LADA in the present study are reliable. This also provides a good opportunity to compare the etiology of LADA and type 2 diabetes. There are also some limitations. First, lacking individual data rules out the possibility of exploring potential nonlinear association between exposures and outcomes, which is a common limitation in MR studies based on summary statistics. The linear assumption is less likely to be violated in our MR study. For LADA, there is no evidence with sufficient statistical power to support a nonlinear association[8, 9]. Adult BMI seemed to be linearly (positively) associated with type 2 diabetes[33, 44]. Observational studies found that the linear (inverse) association between birth weight and type 2 diabetes held when birth weight $<4.0 \mathrm{~kg}[45]$ or $<4.5 \mathrm{~kg}[46]$. The range of birth weight (study-specific: $2.5-4.5 \mathrm{~kg}$ or within the range of mean $\pm 5 \mathrm{SD}$ ) in the GWAS[14] used by the present MR analysis is generally in the range of linear association. Moreover, deviation from the linear assumption is likely to reduce the statistical power in risk estimate, rather than generating spurious associations[47]. Second, findings from the present study were only applicable to the European population since the only GWAS on LADA was conducted in individuals of European ancestry. It is unclear to what extent the findings are generalizable to other populations.

In conclusion, these findings provide genetic support for a causal link between low birth weight, adult overweight/obesity and LADA. The results persisted in a series of sensitivity analyses. Measures should be taken to reduce the prevalence of adult overweight/obesity for 
medRxiv preprint doi: https://doi.org/10.1101/2021.10.25.21265464; this version posted October 25, 2021. The copyright holder for this preprint (which was not certified by peer review) is the author/funder, who has granted medRxiv a license to display the preprint in perpetuity. It is made available under a CC-BY-NC-ND 4.0 International license.

the prevention of diabetes with and without an autoimmune component. The mechanism linking low birth weight to diabetes remains to be explored. 


\section{Acknowledgements}

Not applicable.

\section{Authors' contributions}

YW and SC conceived and designed the study. YW collected the summary data, analyzed data, and drafted the first draft of the manuscript. YZ contributed to methodological issues. All authors critically revised the manuscript for valuable intellectual content. YW and SC contributed to the interpretation of the results. All authors reviewed and approved the final manuscript.

\section{Financial disclosures}

The study was supported by the Swedish Research Council (2018-03035), Research Council for Health, Working Life and Welfare (FORTE, 2018-00337) and Novo Nordisk Foundation (NNF19OC0057274). YW received a scholarship from the China Scholarship Council (student number 202006010041). The sponsors had no role in the study design, data collection, data analysis and interpretation, writing of the report, or the decision to submit the article for publication.

\section{Data availability}

This study only used summary data and these data are publicly available.

\section{Conflict of interest}

None declared. 


\section{References}

[1] Cousminer DL, Ahlqvist E, Mishra R, et al. (2018) First Genome-Wide Association Study of Latent Autoimmune Diabetes in Adults Reveals Novel Insights Linking Immune and Metabolic Diabetes. Diabetes care 41(11): 2396-2403. 10.2337/dc18-1032

[2] Tuomi T, Santoro N, Caprio S, Cai M, Weng J, Groop L (2014) The many faces of diabetes: a disease with increasing heterogeneity. Lancet 383(9922): 1084-1094.

10.1016/S0140-6736(13)62219-9

[3] Bering B, Devendra D (2009) Latent autoimmune diabetes in the young. Clin Med (Lond) 9(1): 93; author reply 93-94. 10.7861/clinmedicine.9-1-93

[4] Yuan S, Larsson SC (2020) An atlas on risk factors for type 2 diabetes: a wide-angled Mendelian randomisation study. Diabetologia 63(11): 2359-2371. 10.1007/s00125-02005253-X

[5] Bellou V, Belbasis L, Tzoulaki I, Evangelou E (2018) Risk factors for type 2 diabetes mellitus: An exposure-wide umbrella review of meta-analyses. PloS one 13(3): e0194127. 10.1371/journal.pone.0194127

[6] Tuomilehto J, Lindström J, Eriksson JG, et al. (2001) Prevention of type 2 diabetes mellitus by changes in lifestyle among subjects with impaired glucose tolerance. $\mathrm{N}$ Engl $\mathbf{J}$ Med 344(18): 1343-1350. 10.1056/nejm200105033441801

[7] Carlsson S (2019) Etiology and Pathogenesis of Latent Autoimmune Diabetes in Adults (LADA) Compared to Type 2 Diabetes. Front Physiol 10: 320. 10.3389/fphys.2019.00320

[8] Hjort R, Ahlqvist E, Carlsson PO, et al. (2018) Overweight, obesity and the risk of LADA: results from a Swedish case-control study and the Norwegian HUNT Study. Diabetologia 61(6): 1333-1343. 10.1007/s00125-018-4596-0 
[9] Hjort R, Alfredsson L, Carlsson PO, et al. (2015) Low birthweight is associated with an increased risk of LADA and type 2 diabetes: results from a Swedish case-control study.

Diabetologia 58(11): 2525-2532. 10.1007/s00125-015-3711-8

[10] Li Y, Ley SH, Tobias DK, et al. (2015) Birth weight and later life adherence to unhealthy lifestyles in predicting type 2 diabetes: prospective cohort study. BMJ 351: h3672.

10.1136/bmj.h3672

[11] Abdullah A, Peeters A, de Courten M, Stoelwinder J (2010) The magnitude of association between overweight and obesity and the risk of diabetes: a meta-analysis of prospective cohort studies. Diabetes Res Clin Pract 89(3): 309-319.

10.1016/j.diabres.2010.04.012

[12] Lawlor DA, Harbord RM, Sterne JA, Timpson N, Davey Smith G (2008) Mendelian randomization: using genes as instruments for making causal inferences in epidemiology. Stat Med 27(8): 1133-1163. 10.1002/sim.3034

[13] Burgess S, Scott RA, Timpson NJ, Davey Smith G, Thompson SG (2015) Using published data in Mendelian randomization: a blueprint for efficient identification of causal risk factors. Eur J Epidemiol 30(7): 543-552. 10.1007/s10654-015-0011-z

[14] Warrington NM, Beaumont RN, Horikoshi M, et al. (2019) Maternal and fetal genetic effects on birth weight and their relevance to cardio-metabolic risk factors. Nature genetics 51(5): 804-814. 10.1038/s41588-019-0403-1

[15] Yengo L, Sidorenko J, Kemper KE, et al. (2018) Meta-analysis of genome-wide association studies for height and body mass index in approximately 700000 individuals of European ancestry. Hum Mol Genet 27(20): 3641-3649. 10.1093/hmg/ddy271

[16] Zhou W, Liu G, Hung RJ, et al. (2021) Causal relationships between body mass index, smoking and lung cancer: Univariable and multivariable Mendelian randomization. Int $\mathbf{J}$ Cancer 148(5): 1077-1086. 10.1002/ijc.33292 
[17] Machiela MJ, Chanock SJ (2015) LDlink: a web-based application for exploring population-specific haplotype structure and linking correlated alleles of possible functional variants. Bioinformatics 31(21): 3555-3557. 10.1093/bioinformatics/btv402

[18] Scott RA, Scott LJ, Mägi R, et al. (2017) An Expanded Genome-Wide Association Study of Type 2 Diabetes in Europeans. Diabetes 66(11): 2888-2902. 10.2337/db16-1253

[19] Burgess; S, Thompson SG (2015) Mendelian Randomization: Methods for Using Genetic Variants in Causal Estimation. CRC Press, 6000 Broken Sound Parkway NW, Suite 300 [20] Burgess S, Butterworth A, Thompson SG (2013) Mendelian randomization analysis with multiple genetic variants using summarized data. Genetic epidemiology 37(7): 658-665. 10.1002/gepi.21758

[21] Slob EAW, Burgess S (2020) A comparison of robust Mendelian randomization methods using summary data. Genetic epidemiology 44(4): 313-329. 10.1002/gepi.22295 [22] Stephen Burgess, Jack Bowden, Frank Dudbridge, Thompson SG (2016) Robust instrumental variable methods using multiple candidate instruments with application to Mendelian randomization. In. Vol 1606.03729. arXiv

[23] Burgess S, Bowden J, Fall T, Ingelsson E, Thompson SG (2017) Sensitivity Analyses for Robust Causal Inference from Mendelian Randomization Analyses with Multiple Genetic Variants. Epidemiology 28(1): 30-42. 10.1097/ede.0000000000000559

[24] Koller M, Stahel WA (2011) Sharpening Wald-type inference in robust regression for small samples. Computational Statistics \& Data Analysis 55(8): 2504-2515.

https://doi.org/10.1016/j.csda.2011.02.014

[25] Bowden J, Davey Smith G, Burgess S (2015) Mendelian randomization with invalid instruments: effect estimation and bias detection through Egger regression. Int J Epidemiol 44(2): 512-525. 10.1093/ije/dyv080 
[26] Bowden J, Davey Smith G, Haycock PC, Burgess S (2016) Consistent Estimation in Mendelian Randomization with Some Invalid Instruments Using a Weighted Median Estimator. Genetic epidemiology 40(4): 304-314. 10.1002/gepi.21965

[27] Verbanck M, Chen CY, Neale B, Do R (2018) Detection of widespread horizontal pleiotropy in causal relationships inferred from Mendelian randomization between complex traits and diseases. Nature genetics 50(5): 693-698. 10.1038/s41588-018-0099-7

[28] Huang T, Wang T, Zheng Y, et al. (2019) Association of Birth Weight With Type 2 Diabetes and Glycemic Traits: A Mendelian Randomization Study. JAMA Netw Open 2(9): e1910915. 10.1001/jamanetworkopen.2019.10915

[29] Zanetti D, Tikkanen E, Gustafsson S, Priest JR, Burgess S, Ingelsson E (2018) Birthweight, Type 2 Diabetes Mellitus, and Cardiovascular Disease: Addressing the Barker Hypothesis With Mendelian Randomization. Circ Genom Precis Med 11(6): e002054. 10.1161/circgen.117.002054

[30] Wang T, Huang T, Li Y, et al. (2016) Low birthweight and risk of type 2 diabetes: a Mendelian randomisation study. Diabetologia 59(9): 1920-1927. 10.1007/s00125-016-4019-z [31] Richardson TG, Sanderson E, Elsworth B, Tilling K, Davey Smith G (2020) Use of genetic variation to separate the effects of early and later life adiposity on disease risk: mendelian randomisation study. BMJ 369: m1203. 10.1136/bmj.m1203 [32] Liu Q, Pan J, Berzuini C, Rutter MK, Guo H (2020) Integrative analysis of Mendelian randomization and Bayesian colocalization highlights four genes with putative BMI-mediated causal pathways to diabetes. Sci Rep 10(1): 7476. 10.1038/s41598-020-64493-4

[33] Wainberg M, Mahajan A, Kundaje A, et al. (2019) Homogeneity in the association of body mass index with type 2 diabetes across the UK Biobank: A Mendelian randomization study. PLoS Med 16(12): e1002982. 10.1371/journal.pmed.1002982 
[34] Shu X, Wu L, Khankari NK, et al. (2019) Associations of obesity and circulating insulin and glucose with breast cancer risk: a Mendelian randomization analysis. Int J Epidemiol 48(3): 795-806. 10.1093/ije/dyy201

[35] Zhu Z, Zheng Z, Zhang F, et al. (2018) Causal associations between risk factors and common diseases inferred from GWAS summary data. Nat Commun 9(1): 224.

$10.1038 / \mathrm{s} 41467-017-02317-2$

[36] Lyall DM, Celis-Morales C, Ward J, et al. (2017) Association of Body Mass Index With Cardiometabolic Disease in the UK Biobank: A Mendelian Randomization Study. JAMA Cardiol 2(8): 882-889. 10.1001/jamacardio.2016.5804

[37] Corbin LJ, Richmond RC, Wade KH, et al. (2016) BMI as a Modifiable Risk Factor for Type 2 Diabetes: Refining and Understanding Causal Estimates Using Mendelian Randomization. Diabetes 65(10): 3002-3007. 10.2337/db16-0418

[38] Holmes MV, Lange LA, Palmer T, et al. (2014) Causal effects of body mass index on cardiometabolic traits and events: a Mendelian randomization analysis. Am J Hum Genet 94(2): 198-208. 10.1016/j.ajhg.2013.12.014

[39] D'Urso S, Wang G, Hwang LD, Moen GH, Warrington NM, Evans DM (2021) A cautionary note on using Mendelian randomization to examine the Barker hypothesis and Developmental Origins of Health and Disease (DOHaD). J Dev Orig Health Dis 12(5): 688693. $10.1017 / \mathrm{s} 2040174420001105$

[40] Hattersley AT, Tooke JE (1999) The fetal insulin hypothesis: an alternative explanation of the association of low birthweight with diabetes and vascular disease. Lancet 353(9166): 1789-1792. 10.1016/s0140-6736(98)07546-1

[41] Barazzoni R, Gortan Cappellari G, Ragni M, Nisoli E (2018) Insulin resistance in obesity: an overview of fundamental alterations. Eat Weight Disord 23(2): 149-157. 10.1007/s40519018-0481-6 
[42] Maddaloni E, Coleman RL, Agbaje O, Buzzetti R, Holman RR (2020) Time-varying risk of microvascular complications in latent autoimmune diabetes of adulthood compared with type 2 diabetes in adults: a post-hoc analysis of the UK Prospective Diabetes Study 30-year follow-up data (UKPDS 86). Lancet Diabetes Endocrinol 8(3): 206-215. 10.1016/s22138587(20)30003-6

[43] Censin JC, Nowak C, Cooper N, Bergsten P, Todd JA, Fall T (2017) Childhood adiposity and risk of type 1 diabetes: A Mendelian randomization study. PLoS Med 14(8): e1002362. 10.1371/journal.pmed.1002362

[44] Narayan KM, Boyle JP, Thompson TJ, Gregg EW, Williamson DF (2007) Effect of BMI on lifetime risk for diabetes in the U.S. Diabetes care 30(6): 1562-1566. 10.2337/dc06-2544 [45] Harder T, Rodekamp E, Schellong K, Dudenhausen JW, Plagemann A (2007) Birth weight and subsequent risk of type 2 diabetes: a meta-analysis. American journal of epidemiology 165(8): 849-857. 10.1093/aje/kwk071

[46] Knop MR, Geng TT, Gorny AW, et al. (2018) Birth Weight and Risk of Type 2 Diabetes Mellitus, Cardiovascular Disease, and Hypertension in Adults: A Meta-Analysis of 7646267 Participants From 135 Studies. J Am Heart Assoc 7(23): e008870. 10.1161/jaha.118.008870 [47] Carreras-Torres R, Johansson M, Gaborieau V, et al. (2017) The Role of Obesity, Type 2 Diabetes, and Metabolic Factors in Pancreatic Cancer: A Mendelian Randomization Study. J Natl Cancer Inst 109(9). 10.1093/jnci/djx012 


\section{Figure Legends}

Figure 1. Flow chart of the study design in the present study

EGG: Early Growth Genetics Consortium; GIANT: Genetic Investigation of

ANthropometric Traits consortium; LADA: latent autoimmune diabetes in

adults; DIAGRAM: the DIAbetes Genetics Replication And Meta-analysis

Consortium.

Figure 2. The risk of LADA in relation to one SD $(0.5 \mathrm{~kg})$ decrease in birth weight

IVW: inverse-variance weighted. MR-Egger: Egger regression of Mendelian randomization;

MR-PRESSO: the Mendelian randomization pleiotropy residual sum and outlier approach;

LADA: latent autoimmune diabetes in adults.

MR-Egger intercept: $-0.012, P$ for directional pleiotropy: 0.443 .

MR-PRESSO detected rs3184504 as the outlier and the outlier was excluded from the outlier corrected estimate ( $P$ for distortion of estimate: 0.457$)$.

\section{Figure 3. The risk of LADA in relation to one $S D\left(4.8 \mathrm{~kg} / \mathrm{m}^{2}\right)$ increase in adult BMI}

IVW: inverse-variance weighted. MR-Egger: Egger regression of Mendelian randomization;

MR-PRESSO: the Mendelian randomization pleiotropy residual sum and outlier approach;

LADA: latent autoimmune diabetes in adults.

The MR-PRESSO identified two outliers: rs11066188 and rs10840606. Outliers were excluded from the outlier-corrected estimate ( $P$ for distortion of estimate: 0.777$)$. 
Table 1. Comparison between LADA and type 2 diabetes

\begin{tabular}{|c|c|c|c|c|c|c|c|}
\hline Exposures & Outcomes & $\begin{array}{l}\text { No. of } \\
\text { initial } \\
\text { SNPs }\end{array}$ & $\begin{array}{c}\text { No. of } \\
\text { outliers }\end{array}$ & $\begin{array}{c}\text { GWAS dataset } \\
\text { for SNP-exposure } \\
\text { association }\end{array}$ & Methods & OR $(95 \% \text { CI })^{b}$ & $\begin{array}{c}P \text { for risk } \\
\text { estimate }\end{array}$ \\
\hline Birth weight & LADA & 129 & 1 & EGG+UK Biobank & MR-PRESSO outlier-corrected & $1.83(1.29-1.59)$ & 0.001 \\
\hline Birth weight & $\begin{array}{c}\text { Type } 2 \\
\text { diabetes }\end{array}$ & 129 & 10 & EGG+UK Biobank & MR-PRESSO outlier-corrected & $1.45(1.25-1.70)$ & $<0.001$ \\
\hline BMI in adulthood & LADA & 734 & 3 & UK Biobank & MR-PRESSO outlier-corrected & $1.42(1.18-1.72)$ & $<0.001$ \\
\hline BMI in adulthood & $\begin{array}{c}\text { Type } 2 \\
\text { diabetes }\end{array}$ & 734 & 9 & UK Biobank & MR-PRESSO outlier-corrected & $2.33(2.16-2.51)$ & $<0.001$ \\
\hline
\end{tabular}

LADA: latent autoimmune diabetes in adults; GWAS: genome-wide association study; EGG: Early Growth Genetics Consortium; IVW: inverse-

variance weighted; MR-PRESSO: the Mendelian randomization pleiotropy residual sum and outlier approach.

${ }^{a}$ Outliers were excluded from the outlier-corrected estimates.

${ }^{\mathrm{b}} \mathrm{OR}(95 \% \mathrm{CI})$ for LADA or type 2 diabetes per SD $(0.5 \mathrm{~kg})$ decrease in birth weight or per SD $\left(4.8 \mathrm{~kg} / \mathrm{m}^{2}\right)$ increase in BMI in adulthood. 
Two-sample Mendelian randomization

Exposures

Instruments

Primary outcome
129 SNPs from EGG + UK Biobank $(n=298140)$
BMI in adulthood
820 SNPs (primary SNPs) from GIANT+UK Biobank $(n=681275)$
734 SNPs (secondary SNPs) from UK Biobank $(n=454884)$
LADA (2634 cases, 5947 controls of European ancestry)

T2D (DIAGRAM: 26676 cases, 132532 controls of of European ancestry) 


\begin{tabular}{|c|c|c|c|c|c|}
\hline Methods & $\begin{array}{l}\text { No. of } \\
\text { SNPs }\end{array}$ & OR (95\% Cl) & & $\begin{array}{c}\text { P for risk } \\
\text { estimate }\end{array}$ & $\begin{array}{l}\text { P for } \\
\text { terogeneity }\end{array}$ \\
\hline IVW & 129 & $2.02(1.37-2.97)$ & $\longrightarrow$ & $<0.001$ & 0.001 \\
\hline Robust IVW & 129 & $1.92(1.37-2.69)$ & $\square$ & $<0.001$ & 0.159 \\
\hline Weighted median & 129 & $1.94(1.17-3.24)$ & $\longrightarrow$ & 0.011 & \\
\hline MR-Egger & 129 & $3.29(0.89-12.08)$ & & 0.073 & 0.001 \\
\hline \multirow[t]{3}{*}{ MR-PRESSO outlier-corrected } & 128 & $1.83(1.29-2.59)$ & $\longrightarrow$ & 0.001 & \\
\hline & & $\Gamma$ & $T$ & 7 & \\
\hline & & 0.50 & $\begin{array}{ccc}1.0 & 2.0 & 5.0 \\
\text { OR }(95 \% & \mathrm{Cl})\end{array}$ & 15.0 & \\
\hline
\end{tabular}




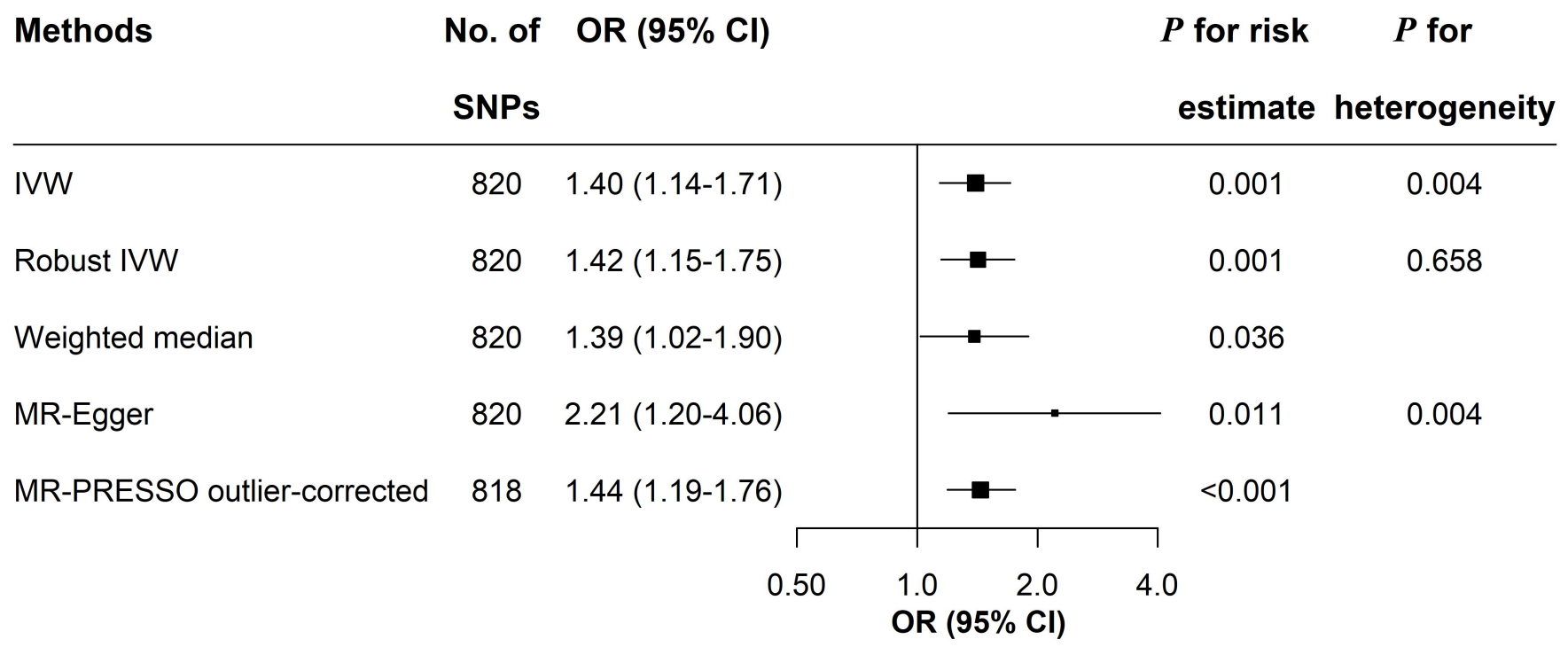

